\title{
La protection du patrimoine religieux en Estonie : lieux de culte et sites naturels sacrés
}

\section{Ringo Ringvee}

\section{(2) OpenEdition}

\section{Journals}

Édition électronique

URL : http://journals.openedition.org/rdr/850

DOI : $10.4000 /$ rdr.850

ISSN : 2534-7462

\section{Éditeur}

Presses universitaires de Strasbourg

Édition imprimée

Date de publication : 3 mai 2017

Pagination : $95-108$

ISBN : 978-2-86820-973-3

ISSN : 2493-8637

\section{Référence électronique}

Ringo Ringvee, «La protection du patrimoine religieux en Estonie : lieux de culte et sites naturels sacrés », Revue du droit des religions [En ligne], 3 | 2017, mis en ligne le 03 février 2020, consulté le 19 novembre 2020. URL : http://journals.openedition.org/rdr/850; DOI : https://doi.org/10.4000/rdr.850

\section{(c) (7) \&}

La revue du droit des religions est mise à disposition selon les termes de la Creative Commons Attribution - Pas d'Utilisation Commerciale 4.0 International - CC BY-NC 4.0. 


\section{LA PROTECTION DU PATRIMOINE RELIGIEUX EN ESTONIE : LIEUX DE CULTE ET SITES NATURELS SACRÉS}

\section{Ringo RINGVEE}

Ministère estonien de l'Intérieur, Département des affaires religieuses

\section{RÉSUMÉ}

Cet article met en lumière certains défis posés au patrimoine religieux estonien, dans le contexte sécularisé du début du $\mathrm{XxI}^{\mathrm{e}}$ siècle. La collaboration entre le gouvernement et l'Église évangélique luthérienne a permis la création de programmes de sauvegarde des édifices du culte ayant une valeur culturelle et historique, malgré des ressources financières limitées. Surtout, il s'agit de montrer comment les sites naturels sacrés sont devenus une question majeure dans le domaine de la protection du patrimoine religieux.

\section{ABSTRACt}

The article pays attention on some of the new challenges for the Estonian religious heritage, in the secularized beginning of the $21^{\text {st }}$ century. Despite limited financial resources, the collaboration between the government and the Evangelical Lutheran Church has resulted in the creation of programs aimed at protecting church buildings of historical and cultural value. Above all, this paper concentrates on the sacred natural sites that have become a key issue in the area of religious heritage protection.

NB. Cette contribution correspond pour partie à la mise à jour et traduction d'un article paru dans Fornerod A. (ed.), Funding religious heritage, Farnham, Ashgate, 2015. 
T a protection et la conservation du patrimoine culturel estonien sont —étroitement liées à l'histoire, et plus précisément aux épisodes de guerre. Avec la guerre d'indépendance de 1918-1920 apparaît l'attention portée au patrimoine, qui se caractérise dès cette époque comme une initiative civique ${ }^{1}$. En 1919, alors que les conflits opposent l'Estonie à la Russie bolchevique à l'est et aux corps francs allemands au sud, le comité central estonien des sociétés culturelles établit deux comités de protection des arts afin de veiller à la préservation des monuments culturels ${ }^{2}$.

Le premier texte légal régissant la protection du patrimoine, la loi sur la protection des antiquités, fut adopté en $1925^{3}$. Une version modifiée de cette loi fut appliquée à partir de 1936, alors qu'était nommé le premier inspecteur des monuments historiques, dépendant du gouvernement ${ }^{4}$. La loi ne distinguait pas entre les monuments religieux et les autres. Dans les années 1920 et encore dans les années 1930, les recherches et la protection se concentraient sur les débuts de l'histoire estonienne et le Moyen Âge, tandis que les premiers manoirs de la Baltique allemande furent classés comme monuments culturels dans les années $1930^{5}$. Les batailles de la Seconde Guerre mondiale opposant les troupes soviétiques et allemandes traversèrent l'Estonie en 1941 et 1944, avec des effets dévastateurs sur le patrimoine culturel. Ainsi, les batailles de 1944 détruisirent complètement Narva, unique ville baroque du $\mathrm{XVII}^{\mathrm{e}}$ siècle, mais aussi la moitié du centre-ville de Tartu et une partie de la vieille ville de l'époque médiévale de la capitale Tallinn. Les édifices religieux furent sévèrement endommagés à travers tout le pays.

L'annexion de l'Estonie par l'Union soviétique en 1940 se traduisit par l'adoption d'une législation sur la conservation et l'entretien des monuments historiques, qui s'appliqua à partir de 1947. La loi sur la conservation des

1. À cette époque, et depuis lors, la recherche dans le domaine du patrimoine culturel, mais aussi le processus de sélection et la protection procèdent du bénévolat et sont le fait, essentiellement, de professeurs et d'étudiants de l'Université de Tartu.

2. RaAl M., « Kunstiväärtuste kaitsmine Eestis 1919-1921 » [Protection des biens artistiques en Estonie 1919-1921], in Randla A. (ed.), Mälu. Eesti Kunstiakadeemia Toimetised 20, Tallinn, Estonian Academy of Arts, 2011, p. 149 et 152.

3. Muinasvarade kaitse seadus [Loi sur la conservation des antiquités], Riigi Teataja [Journal officiel], 1925, 111/112.

4. Muinasvarade kaitse seadus [Loi sur la conservation des antiquités], Riigi Teataja, $1936,67$.

5. Alatalu R., Muinsuskaitse siirdeühiskonnas 1986-2002 : rahvuslikust südametunnistusest Eesti NSV-somaniku ahistajaks Eesti Vabariigis [Le patrimoine dans une société transitionnelle, 1986-2002 : de la conscience nationale dans la République socialiste soviétique d'Estonie au harcèlement du propriétaire privé en République d'Estonie], Tallinn, Eesti Kunstiakadeemia, 2012, p. 41. 
monuments culturels dans la République socialiste soviétique d'Estonie qui fut adoptée en 1961 était la première de ce type en Union Soviétique ${ }^{6}$. Les spécialistes en la matière qui œuvraient déjà pendant la période d'indépendance élaborèrent ce texte selon les principes expérimentés dans les années 1920 et $1930^{7}$. Les premières dispositions adoptées par le gouvernement central d'URSS entrèrent en vigueur en 1977. La restauration et la conservation professionnelles des édifices religieux en Estonie pendant la période soviétique débutèrent cependant dès les années 1950 et furent approfondies dans les années $1980^{8}$. Certains de ces édifices furent alors considérés comme des monuments d'architecture ou culturels et, à ce titre, protégés de la même façon que les autres monuments. La loi de 1977 avait tenu compte de l'engagement de nature civique dans la protection du patrimoine culturel et, en conséquence, des sociétés locales du patrimoine culturel s'établirent en rassemblant les jeunes générations. Près d'une décennie plus tard, ces clubs du patrimoine formèrent un forum politique majeur pour le soutien du réveil national ${ }^{9}$.

L'Estonie a donc hérité du siècle dernier un dispositif législatif relatif au patrimoine culturel et historique applicable au patrimoine religieux et partage avec de nombreux autres pays européens un contexte sociétal marqué par la sécularisation (1). Au Xxi ${ }^{e}$ siècle, l'État estonien et les groupes religieux sont confrontés à de nouveaux défis en matière de protection du patrimoine religieux. Sur un plan strictement technique, cela concerne par exemple l'usage de nouveaux matériaux de conservation des objets protégés, le contexte de sécularisation n'empêchant pas une attention soutenue portée au patrimoine religieux (2). D'autres défis soulèvent des questions plus profondes sur le sacré, comme l'illustrent les débats sur l'essence et les frontières des sites naturels sacrés et leurs conséquences juridiques (3).

6. Eesti NSV seadus Eesti NSV kultuurimälestiste kaitse kohta [Loi de la République socialiste soviétique d'Estonie sur la conservation des monuments culturels], Eesti NSV Ülemnõukogu Teataja [Bulletin de la Cour suprême d'Estonie], 23, 15.06.1961.

7. Alatalu R., op. cit., p. 42.

8. Ibid., p. 118.

9. Kasekamp A., A History of the Baltic States, Basingstoke, Palgrave Macmillan, 2010, p. 161. - Rohtmets P., Ringvee R., «Religious Revival and the Political Activity of Religious Communities in Estonia during the Process of Liberation and the Collapse of the Soviet Union 1985-1991 », Religion, State and Society, 2013, 41 (4), p. 355-393. 


\section{LE CADRE INSTITUTIONNEL ET LÉGISLATIF APPLICABLE AU PATRIMOINE RELIGIEUX}

\subsection{PROTÉGER LE PATRIMOINE CULTUREL : L'HÉRITAGE DU XXE SIÈCLE}

La protection du patrimoine était devenue un enjeu de taille dans le cadre du réveil national à la fin des années 1980 et l'élaboration d'une nouvelle législation relative à la conservation du patrimoine débuta en 1990. Ce processus aboutit en 1994 à l'adoption d'une première loi sur la conservation du patrimoine ${ }^{10}$, suivie d'une nouvelle version du texte en 2002, elle-même modifiée à plusieurs reprises ${ }^{11}$. La loi ne fait pas de distinction entre monuments régionaux et nationaux, ni ne réserve de catégorie spécifique pour les biens religieux. Selon cette loi, un édifice peut être classé en tant que monument archéologique, architectural, artistique, technique, industriel ou historique. L'article 2 de la loi sur la conservation du patrimoine définit le monument culturel comme : «Un bien meuble ou immeuble, en tout ou partie, un ensemble de biens ou de structures sous la protection de l'État, ayant un intérêt historique, archéologique, ethnographique, de développement urbain, architectural, artistique ou scientifique ou un intérêt en termes d'histoire religieuse ou culturelle. »

En application de cette loi, les biens immobiliers incluent les édifices du culte, les cimetières, les pierres à cupules et les édifices sacrés présentant une valeur artistique ou un intérêt au regard de l'histoire culturelle ${ }^{12}$. Les biens mobiliers incluent les objets ethnographiques et les ouvres d'art visuel et appliqué ayant un intérêt du point de vue de l'histoire culturelle ou religieuse.

Les années 1990 furent, tant pour la protection du patrimoine que pour la société dans son ensemble, une période de transition mais également le moment de déterminer des priorités. En conséquence, au début des années 2000, plusieurs programmes dans le domaine de la conservation du patrimoine purent être initiés, dont le premier, en 2001, désigné comme le programme «Écoles dans les manoirs anciens - préservation d'ensembles historiques et culturels et transformation en centres d'apprentissage contemporains ». Ce programme visait à transformer les écoles situées dans d'anciens manoirs en centres régionaux d'éducation et de culture modernisés, tout en s'assurant

10. Muinsuskaitse seadus [Loi sur la conservation du patrimoine], Riigi Teataja, RT I 1994, 24, 391.

11. Muinsuskaitse seadus [Loi sur la conservation du patrimoine], Riigi Teataja, RT I 2002, 47, 297.

12. En 2012, l'Estonie comptait 26076 monuments classés. V. Alatalu R., op. cit., p. 183. 
que ces bâtiments historiques et le mobilier protégé qu'ils contiennent fussent préservés. Ce programme fut suivi en 2003 par un programme d'État pour la conservation des édifices du culte et un autre consacré à l'architecture et aux paysages ruraux, en 2007.

\subsection{LE PATRIMOINE RELIGIEUX ENTRE INSTITUTIONNALISATION ET SÉCULARISATION}

Les changements sociopolitiques intervenus à partir des années 1980 culminèrent avec le retour de l'indépendance de l'Estonie en 1991, consacré par une nouvelle Constitution adoptée par référendum en 1992. La liberté de religion et le principe de l'absence d'Église d'État en Estonie sont énoncés à l'article 40 de la Constitution de 1992. C'est au cours de cette période que la religion et les institutions religieuses revinrent dans la sphère publique et que les relations entre l'État et les groupes religieux se restructurèrent, en particulier à travers l'adoption de la loi sur les Églises et les congrégations (Kirikute ja koguduste seadus) en $1993^{13}$. Cette loi établissait une situation de marché néolibéral en matière religieuse ${ }^{14}$.

Le modèle de relations État-religions qui en découle pourrait être qualifié de système de coopération dans les domaines d'intérêt commun, dont fait partie la protection du patrimoine ${ }^{15}$. Les principaux interlocuteurs de l'État sont l'Église évangélique luthérienne d'Estonie (ci-après EELE) et le Conseil estonien des Églises (ci-après CEE), lequel, établi en 1989, constitue une organisation-cadre regroupant les Églises chrétiennes estoniennes et les associations de congrégations (communautés paroissiales locales). Le CEE représente l'organisation œcuménique la plus importante d'Estonie ${ }^{16}$. En

13. Kirikute ja koguduste seadus [Loi sur les Églises et les congrégations], Riigi Teataja, RT I 1993, 30, 510. La loi de 1993 fut remplacée par un nouveau texte en 2002 : Kirikute ja koguduste seadus [Loi sur les Églises et les congrégations], Riigi Teataja, RT I 2002, 24, p. 135, disponible en ligne en version anglaise : https://www.riigiteataja.ee/en/ eli/523012015005/consolide [consulté le 13 janv. 2017]. V. KIVIORG M., Religion and Law in Estonia, Alphen aan den Rijn, Kluwer, $2^{\text {nd }}$ ed. 2016.

14. RingVEE R., Riik ja religioon nõukogudejärgses Eestis 1991-2008 [État et religion en Estonie post-soviétique 1991-2008, résumé en anglais], Tartu University Press, 2011.

15. Ibid., p. 91-92.

16. Si l'on tient compte des recensements, les associations membres du CEE représentent plus de $90 \%$ de la population ayant une affiliation religieuse. Actuellement, le CEE inclut l'Église évangélique luthérienne, l'Église apostolique orthodoxe, l'Église orthodoxe du patriarcat de Moscou, l'Église catholique romaine, l'Union des Églises chrétiennes évangéliques et baptistes, l'Église méthodiste, l'Association des adventistes du septième jour, l'Église chrétienne pentecôtiste, une congrégation de l'Église apostolique arménienne et l'Église épiscopale charismatique d'Estonie. 
2002, le gouvernement estonien signa un protocole d'intérêts communs avec le CEE. Le ministère de l'Intérieur et le CEE réitérèrent les principes fondant leur coopérant établis en 2002 par un nouvel accord en 2015 ${ }^{17}$. Les raisons d'un tel accord entre État et religion relèvent du pragmatisme : l'EELE est l'Église majoritaire depuis le $\mathrm{XVI}^{\mathrm{e}}$ siècle, dotée historiquement d'une forte influence sur la culture estonienne, et les congrégations luthériennes couvrent tout le territoire de l'Estonie. En 1995, le gouvernement établit un comité mixte avec l'EELE, comprenant une sous-commission pour le patrimoine architectural. Il est ici à signaler que la majorité des 219 édifices cultuels classés comme monuments nationaux sont la propriété de congrégations de l'EELE, tandis que l'Église orthodoxe estonienne du patriarcat de Moscou et les Vieux-croyants sont propriétaires respectivement de treize et deux édifices classés monuments historiques.

Selon différentes études et sondages, l'Estonie du Xxi siècle est l'une des sociétés européennes ayant le plus bas taux de fréquentation des églises et l'un des rares pays où la majorité de la population n'a pas d'affiliation religieuse. La sécularisation de la société estonienne se traduit donc par une faible fréquentation hebdomadaire des services religieux. Selon le recensement de la population de 2011, $29 \%$ de la population de plus de quinze ans s'estiment affiliés à une tradition religieuse ${ }^{18}$. Quatre ans plus tard, 4,7\% des sondés déclaraient assister à un office religieux au moins une fois par semaine. La même année, une étude indiquait que, dans l'ordre des motivations pour assister à ces offices, la qualité de l'architecture de l'édifice cultuel venait en quatrième position ${ }^{19}$, illustrant le fait que la sécularisation peut s'associer à la patrimonialisation.

L'on observe une autre conséquence de la sécularisation s'agissant de la dimension financière de la gestion du patrimoine religieux. En effet, les

17. Eesti Vabariigi Valitsuse ja Eesti Kirikute Nõukogu Ühishuvide Protokoll, 17.10.2002 [Protocole d'intérêts communs entre le gouvernement de la République d'Estonie et le Conseil estonien des Églises] ; Siseminsiteeriumi ja Eesti Kirikute Nõukogu koostööleping, 21.01.2015 [Accord de coopération entre le ministère de l'Intérieur et le Conseil estonien des Églises].

18. Estonian Statistics. Statistical Database: Population and Housing Census 2011. Demographic and ethno-cultural characteristics of the population. Religious Affiliation.

19. Étude Elust, usust ja usuelust 2015 [La vie, la foi et la vie religieuse en 2015], non publiée. Les données issues du recensement ainsi que d'autres études montrent une différence considérable entre les Estoniens et les personnes appartenant aux minorités ethniques dans leur relation à la religion. Selon le recensement, $55 \%$ des personnes ayant une affiliation religieuse font partie des minorités ethniques. Selon une étude de 2015, $18 \%$ des Estoniens se considèrent comme membre d'associations religieuses, le pourcentage étant de $25 \%$ pour les personnes issues des minorités ethniques. 
associations religieuses estoniennes s'autofinancent : leur budget dépend des dons et de leurs activités économiques qui demeurent rares. Le vieillissement et la diminution des membres des congrégations se sont révélés problématiques, spécialement pour l'EELE, propriétaire du plus grand nombre d'édifices religieux, dont la majorité nécessite des travaux de conservation et de restauration. La diminution des dons des membres pose nécessairement des limites à la réalisation de tels travaux ${ }^{20}$ et implique de recourir aux subventions étatiques ou municipales.

Dans ce contexte, le programme gouvernemental « Conservation et développement des lieux de culte » appelle quelques développements.

\section{LE PROGRAMME « CONSERVATION ET DÉVELOPPEMENT DES LIEUX DE CULTE ॥}

Le programme gouvernemental «Conservation et développement des lieux de culte », adopté en 2003 et étendu jusqu'en 2018 ${ }^{21}$, vise à conserver et restaurer les édifices du culte ayant une valeur culturelle et historique, ainsi que les cimetières et les objets sacrés (autels, icônes...). L'idée d'introduire un tel programme revient au comité mixte précité, créé en 1995 et associant l'EELE et le gouvernement. Au ministère de la Culture, le Comité national du patrimoine est responsable de la mise en œuvre et du pilotage du programme. Le budget alloué couvre la conservation et la restauration des biens concernés mais sert également au financement de projets autour de ce patrimoine comme à l'installation d'alarmes de sécurité ou de paratonnerres ou encore aux recherches scientifiques le concernant. Il est à souligner que ce programme ne s'applique pas qu'aux seuls édifices et objets classés : un cinquième du budget a été utilisé pour la conservation de lieux de culte non protégés au titre des monuments historiques.

La majorité des édifices et objets religieux monuments historiques sont la propriété ou sont utilisés par l'EELE, ce qui se reflète dans la répartition de la dotation du programme : entre 2004 et 2010, l'EELE en a reçu $71,3 \%$

20. NitT R., "The State of Church Buildings in Estonia. Good Economic Management of a Church », in Hansson J., Ranta H. (eds), Archaeology and History of Churches in Baltic Region, Visby, Gotland University Press, 2011, p. 233-236.

21. Riiklik programm 'Pühakodade säilitamine ja areng' [Programme gouvernemental « Conservation et développement des lieux de culte»], consultable sur le site du Comité national du patrimoine : http://www.muinas.ee/puhakoda [consulté le 13 janv. 2017]. Le site contient notamment les budgets annuels du programme. 
(4 654000 euros) ${ }^{22}$. Cette tendance a toutefois évolué ces dernières années, les subventions versées à l'EELE n'atteignant plus que $40 \%$ du budget total. En 2016, sept mouvements religieux ont bénéficié d'un financement à travers ce programme ${ }^{23}$. À l'instar d'autres projets de ce type, il subsiste toujours un décalage entre les besoins et les ressources financières. La plupart des travaux entrepris dans les édifices religieux et financés par le programme sont réalisés dans l'urgence. Il n'en reste pas moins qu'il s'agit là d'un cadre de collaboration fructueuse entre le Comité national du patrimoine et les institutions de recherche comme l'Université technique de Tallinn et l'Académie des arts d'Estonie. Cette coopération a donné lieu à des publications sur trois édifices religieux, sur des intérieurs d'églises et à la création d'archives numériques sur la conservation des églises ${ }^{24}$.

D'autres programmes portent sur le patrimoine religieux, aux niveaux régional et municipal. Ainsi, le programme d'ampleur concernant les églises de la Renaissance de Tallinn (2002-2011) a permis de financer la conservation de vingt-deux églises de la ville. Il est d'ailleurs d'usage courant en Estonie que les autorités locales offrent leur concours financier pour la conservation des édifices cultuels. En 2008, le ministre des Affaires régionales, auprès du ministère de l'Intérieur, a approuvé un programme pour la préservation des traditions des Vieux-croyants installés sur la côte ouest du lac Peippous, qui a permis le financement de la conservation et de la restauration de leurs églises, icônes et manuscrits. De manière générale, de nombreux monuments ont reçu des subventions directes de l'État ou de l'autorité locale pour leur conservation. Parmi les multiples exemples qui pourraient être donnés, méritent d'être citées l'église médiévale Saint-Jean à Tartu et l'église estonienne luthérienne Saint-Jean à Saint-Pétersbourg en Russie ${ }^{25}$.

Un des points problématiques soulevé par la protection du patrimoine résulte de la rencontre entre les principes guidant la conservation et une lecture religieuse des monuments qui peut entrer en opposition avec les règles

22. Uustalu K., «Le financement des lieux de culte par l'État et les autorités locales en Estonie ", intervention lors de la Conférence sur les valeurs chrétiennes, Tallinn, 19 nov. 2010 .

23. Il s'agit des luthériens, des deux Églises orthodoxes, des baptistes, des méthodistes, de l'Église des Frères moraves et des Vieux-croyants russes.

24. V. http://kirikud.muinas.ee [consulté le 13 janv. 2017]. V. aussi Uustalu K., précit.

25. En 2014, l'Estonie obtint l'édifice historique de l'église Saint-Jean et tandis que l'EELE assure les services religieux en Estonie, le bâtiment est géré par la Fondation Eesti Kontsert et est considéré comme une ambassade culturelle d'Estonie informelle dans la ville, dans la mesure où il sert de salle de concert. V. http://www.concert.ee/index. php? sisu=esileht\&mid = 123\&lang = eng [consulté le 13 janv. 2017]. V. Alatalu R., op. cit., p. 118-119. 
régissant cette conservation. Les difficultés surgissent lorsque de nouveaux matériaux sont utilisés uniquement selon des considérations esthétiques, en négligeant voire ignorant les principes de la conservation. Ce fut le cas en 2005 lorsqu'une congrégation entreprit, en toute bonne foi, de peindre un autel du XVII siècle et une chaire avec de vives couleurs synthétiques, qui suscitèrent l'approbation et l'enthousiasme du pasteur et de la congrégation mais endommagèrent sérieusement les biens ${ }^{26}$. C'est à la suite de cet incident qu'en 2006 le Comité national pour le patrimoine et le ministère de l'Intérieur initièrent un programme d'information sur l'entretien des édifices et objets sacrés, à l'intention du clergé et des représentants des congrégations. Ce programme de sensibilisation aux règles de la conservation des monuments est devenu régulier et s'adresse aux différentes confessions. Cette initiative a rencontré un succès certain et a donné lieu à la publication d'un guide sur la bonne conservation des édifices religieux ${ }^{27}$.

\section{LA PROTECTION DES SITES NATURELS SACRÉS}

\subsection{LE PLAN DE DÉVELOPPEMENT POUR LA PROTECTION DES SITES NATURELS SACRÉS HISTORIQUES}

Au début du xxI $^{\mathrm{e}}$ siècle, l'association locale des païens autochtones, la Maison de Taara et des religions autochtones (Taarausuliste ja Maausuliste Maavalla Koda, ci-après la MTRA) ${ }^{28}$, devint une voix critique des politiques

26. Registre national des monuments culturels, monument 15373, église de Vigala : http:// register.muinas.ee/?menuID=monument\&action = view\&id = 15373 [consulté le 13 janv. 2017].

27. L'ouvrage est disponible sur le site du Comité national du patrimoine en estonien (http:// www.muinas.ee/files/Kirikute\%20hooldusraamat_pdf) ainsi qu'en russe (http://www. muinas.ee/files/Kirikute\%20hooldusraamat\%20RUS_.pdf) [consultés le 13 janv. 2017].

28. La Maison de Taara et des religions autochtones fut créée en 1995 afin de représenter deux mouvements de paiens autochtones contemporains : la religion de Taara et Maausk. La première fut fondée dans les années 1920 comme un mouvement autochtone néopaien. La seconde, Maausk (littéralement « religion de la Terre»), émergea durant le réveil national à la fin des années 1980 et était liée au mouvement folklorique des années 1960 (V. VAKKER T., « Rahvusliku religiooni konstrueerimise katsed 1920-1930 aastate Eestis - taara usk» [Les efforts de construction d'une religion nationale en Estonie dans les années 1920-1930 - La croyance de Taara, résumé en anglais], Mäetagused, 2012, 50 : http://www.folklore.ee/tagused/nr50/vakker.pdf [consulté le 13 janv. 2017] ; V. aussi VÄstRIK E.-H., «In Search of Genuine Religion : The Contemporary Estonian Maausulised Movement and National Discourse », in Rountree K. (ed.), Contemporary Pagan and Native Faith Movements in Europe : Colonialist and Nationalist Impulses, Oxford, Berghahn, 2015, p. 130-153. 
gouvernementales sur la religion, car considérées comme trop orientées vers le christianisme ${ }^{29}$. Leurs reproches portaient sur la coopération entre l'État et les Églises chrétiennes dans le domaine de l'assistance spirituelle dans les forces armées et les prisons et de l'éducation religieuse dans les écoles publiques, qui équivalaient à leurs yeux à financer une mission chrétienne $e^{30}$. La MTRA défendait également l'idée que la conservation des édifices religieux revenait à financer indirectement le christianisme ${ }^{31}$. Toutefois, peu de temps après l'adoption du programme "Conservation et développement des lieux de culte », les premières initiatives en faveur d'un programme similaire pour la protection des lieux et sites naturels sacrés historiques vinrent de la MTRA. Des archéologues et ethnologues de l'Université de Tartu soutinrent l'idée d'un programme consacré spécifiquement aux sites sacrés et obtinrent une réponse positive du Comité national du patrimoine. En 2005 se tint à Tallinn la première conférence sur les sites naturels sacrés, dont les actes furent publiés par la Société savante estonienne (Õpetatud Eesti Selts) ${ }^{32}$.

En septembre 2006, le ministère de la Culture organisa la première table ronde sur les lieux saints naturels et étudia alors les possibilités d'un plan d'engagement gouvernemental et de mesures de conservation. À la suite de cette table ronde, il installa un comité de travail chargé de préparer un programme, qui réunissait des représentants de différents ministères et institutions publiques ainsi que des représentants des groupes religieux et du monde universitaire. Le ministre de la Culture signa le plan de développement

À l'instar d'autres États balkaniques, le mouvement folklorique estonien soulignait l'importance du folklore local et des chants traditionnels et également le lien avec d'autres nations finno-ougriennes (V. Smidchens G., A Baltic Music: The Folklore Movement in Lithuania, Latvia, and Estonia, 1968-1991, thèse, Université de l'Indiana, 1996 : https:// scholarworks. iu.edu/dspace/handle/2022/20479 [consulté le 13 janv. 2017]. Ces dernières années, la Maausk est devenue la branche dominante au sein de la MTRA. V. aussi RingVeE R., « New Religious Movements and New Age in Estonia », in LEWIS J.R., BÅRDSEN Tøllefsen I. (eds), Handbook of Nordic New Religions, Leiden, Brill, 2015, p. 478-494.

29. Vakker T., Rohtmets P., «Estonia : Relations between Christian and Non-Christian Religious Organisations and the State of Religious Freedom », Religion, State and Society, 2008, 36 (1), p. 48-50.

30. La liste des déclarations officielles de la MTRA sur différentes questions est disponible - en estonien - sur le site de la MTRA : http://www.maavald.ee [consulté le 13 janv. 2017].

31. V. Lipp K., «Ahto Kaasik: Eestil on praktilise usuvabaduseni veel pikk tee minna (I osa)» [Ahto Kaasik: la longue route de l'Estonie vers une liberté de religion effective. $1^{\text {re }}$ partie], 2008 : www.bioneer.ee/bioneer/arvamus/aid-1552/AHTO-KAASIK\%3A-Eestilon-praktilise-usuvabaduseni-veel-pikk-tee-minna-I-osa- [consulté le 13 janv. 2017].

32. Valk H. (ed.), Looduslikud pühapaigad. Väärtused ja kaitse [Sites naturels sacrés. Valeurs et conservation], Tartu, Õpetatud Eesti Selts, 2007, résumé en anglais : www.muinas.ee/ files/Looduslikud\%20pyhapaigad.pdf [consulté le 13 janv. 2017]. 
pour la protection des sites naturels sacrés historiques en $2008^{33}$. L'initiative de la MTRA faisait écho à l'identité estonienne dans son lien avec la nature et avait rencontré un soutien politique, conduisant ainsi à une formalisation à travers ce projet consacré aux sites naturels sacrés. Ce programme a bénéficié d'un soutien financier substantiel sur le budget de l'État de l'année 2008, à l'initiative du Parti vert estonien ${ }^{34}$. Ainsi, 1,5 million de couronnes estoniennes (environ 100000 euros) furent alloués, sur le budget de l'État, à la Fondation Hiite Maja (la Maison des bosquets), créée par la MTRA, en vue de la mise en ouvre du plan de développement ${ }^{35}$.

Le plan de développement "Lieux saints naturels historiques en Estonie », pour la période 2008-2012, a défini les lieux sacrés comme " historiques » dès lors que leur histoire remonte à plus de cent ans et que cette historicité s'appuie sur des archives ou d'autres sources ${ }^{36}$. Le plan de développement se concentrait sur les sites sacrés historiques naturels (les bosquets, les sources, les arbres, pierres, etc.) considérés comme formant une part importante du patrimoine estonien, mais également de l'identité nationale, en lien étroit avec les forêts où les Estoniens avaient trouvé abri durant les guerres et les persécutions. L'Estonie compterait ainsi approximativement 2800 sites naturels sacrés historiques, le chiffre exact demeurant discuté. À l'instar du programme précité pour les lieux de culte, le programme consacré aux sites naturels a été touché par un manque de moyens financiers ${ }^{37}$.

Alors que le plan de développement pour les sites sacrés prenait fin en 2012, des tensions apparurent entre les différentes parties prenantes, quant à la méthodologie employée mais également quant à l'objectif général de conservation des sites sacrés, retardant l'adoption d'une suite au plan de développement. C'est donc après des discussions au sein du comité de travail

33. Ministri käskkiri 01. Aprill 2008 nr 146. Valdkonna arengukava 'Eesti ajaloolised looduslikud pühapaigad. Uurimine ja hoidmine' aastateks 2008-2012 kinnitamine [Ministre de la Culture, décret $n^{\circ} 146 \mathrm{du} 1^{\mathrm{er}}$ avril 2008 portant approbation du plan de développement «Sites naturels sacrés historiques d'Estonie, 2008-2012»].

34. Le Parti vert estonien (également appelé les Verts estoniens, Erakond Eestimaa Rohelised) était représenté au Parlement de 2007 à 2011. Bien que ne faisant pas partie de la majorité gouvernementale, ils eurent un poids décisif dans certains votes.

35. Compte-rendu du Comité des finances du Parlement (Riigikogu), n 152, 7 déc. 2008 : http://web.riigikogu.ee [consulté le 13 janv. 2013]

36. Valdkonna arengukava 2008-2012 'Eesti ajaloolised looduslikud pühapaigad. Uurimine ja hoidmine' [Plan de développement « Lieux saints sacrés historiques en Estonie. Étude et préservation, 2008-2012»].

37. KAASIK A., Eesti ajaloolised looduslikud pühapaigad. Uurimine ja hoidmine. Valdkonna arengukava 2008-2012. Koondaruanne [Lieux saints sacrés historiques en Estonie. Étude et préservation, 2008-2012. Rapport résumé], Université de Tartu, 2013, p. 7. 
qu'un nouveau plan de développement «Sites naturels sacrés estoniens. Recherche et conservation 2015-2020 » fut finalement signé par le ministre de la Culture en 2015. Si les discussions ont donné lieu à des débats sur le financement et la méthodologie (par exemple sur la prise en compte du folklore contemporain lié aux sites naturels sacrés lors des inventaires), l'objectif général du plan de développement demeure le même ${ }^{38}$.

\subsection{LE DÉFI DE LA PROTECTION DES SITES NATURELS SACRÉS}

Le respect et la déférence à l'égard de la nature occupent une place importante dans la tradition populaire estonienne. Les traditions religieuses autochtones préchrétiennes sont généralement analysées comme une vénération animiste de la nature et leurs sites sacrés sont installés dans la nature depuis des siècles. Certaines de ces traditions ont été préservées jusqu'à aujourd'hui, telles les croix gravées sur les arbres lors des funérailles dans certaines parts du sud-est de l'Estonie, mais également, et plus généralement, les lieux saints ou sacrés dans la nature (arbres, bosquets, sources, pierres) qui sont toujours en usage aujourd'hui ${ }^{39}$.

Certains de ces sites sacrés furent protégés dès les années 1920, après l'accession à l'indépendance de l'Estonie et ce processus continua pendant la période soviétique. Aujourd'hui, ces sites sacrés sont protégés sur le fondement de deux lois différentes : la loi sur la conservation du patrimoine ${ }^{40}$ et la loi sur la conservation de la nature ${ }^{41}$. Il en résulte que les sites et lieux sacrés peuvent relever de différentes catégories visant à leur protection. Ainsi, en 2016, l'on comptait 455 sites naturels sacrés protégés, certains de ces sites

38. Texte du plan de développement 2015-2020 disponible en ligne (en estonien) : www. muinas.ee/files/ALPAK\%20arengukava_2015-2020.docx [consulté le 13 janv. 2017].

39. V. Jonuks T., "From Holy Hiis to Sacred Stone : Diverse and Dynamic Meanings of Estonian Holy Sites », in Rountree K., Morris C., Peatfield A. (eds), Archaeology of Spiritualities, New York, Springer, 2012, p. 163-183. - Jonuks T., Veldi M., Oras E., «Looduslikud pühapaigad - uue ja vana piiril» [Les sites naturels sacrés - nouvelle et ancienne frontière], Vikerkaar, 2014, p. 93-108. - KõıvUPuU M., « Natural sacred places in landscape : an Estonian mode », in Bergmann S., Scott P.-M., Jansdotter SAmuelsson M. and Bedford-Strohm H. (eds), Nature, Space and the Sacred. Transdisciplinary Perspectives, Farnham, Ashgate, 2009, p. 223-234. - VAlK H., « Sacred Natural Places of Estonia : Regional Aspects », Folklore. Electronic Journal of Folklore, 2009, vol. 42 : www.folklore. ee/folklore/vol42/valk.pdf [consulté le 13 janv. 2017].

40. Muinsuskaitse seadus [Loi sur la conservation du patrimoine], Riigi Teataja, RT I, 23.03.2015, 128. Traduction anglaise disponible en ligne : https://www.riigiteataja.ee/ en/eli/517062015012/consolide [consulté le 13 janv. 2017].

41. Looduskaitse seadus [Loi sur la conservation de la nature], Riigi Teataja, RT I 2004, $38,258$. 
- et objets - étant protégés pour leur valeur culturelle, d'autres parce qu'ils constituent d'importants sites archéologiques, d'autres encore en ce qu'ils permettent de préserver en tout ou partie des zones naturelles d'une valeur culturelle ou esthétique.

Il n'en reste pas moins que $80 \%$ des sites et objets sacrés naturels seraient sans protection ${ }^{42}$. En outre, plusieurs sites protégés n'ont pas de limites fixes ou leur délimitation est l'objet de discussions. La MTRA et la Maison des bosquets ont formé plusieurs recours en justice afin de s'opposer à des activités économiques réalisées sur des sites sacrés en violation des principes applicables à la gestion de ces sites ${ }^{43}$. De manière générale, la difficulté vient de ce que l'existence de preuves - et leur fiabilité - que ces lieux ont été des sites sacrés historiques fait débat.

Par ailleurs, la mise en ouvre du plan de développement pour la conservation des sites naturels sacrés soulève de nouveaux questionnements pour le législateur. À la différence des édifices cultuels qui sont la propriété des associations religieuses, ou sont utilisés par elles, et qui servent à des fins de célébration du culte, aucun de ces sites sacrés historiques n'est la propriété d'associations religieuses. En revanche, tout comme la conservation des édifices religieux et le classement au titre des monuments historiques entraînent l'application de règles spécifiques et des limitations aux droits du propriétaire, la protection des sites naturels sacrés se traduit par des limites aux activités économiques du propriétaire. Sur ce point, les différents intérêts en présence peuvent aisément se heurter, comme le montrent les recours judiciaires relatifs à la protection de ces sites. La MTRA et la fondation Maison des bosquets insistèrent au cours de la période 2008-2012 sur la nécessité d'une législation spécifiquement consacrée à la protection des sites naturels sacrés historiques, mais sans résultat.

Dans ce cadre de réflexion, la question a été soulevée de la protection des sites sacrés non pas seulement en tant qu'objets matériels, mais aussi comme espaces spirituels. Ici, la question déjà délicate d'une délimitation des sites sacrés clairement établie dans les documents cadastraux en vue de leur protection se complexifie devant la perspective de les protéger également en

42. KAASIK A., op. cit., p. 5.

43. Ces recours ont connu des issues variables. En 2007, la MTRA a obtenu gain de cause contre le développement d'un parc éolien sur une colline sacrée dans le nord-est de l'Estonie, tandis qu'un jugement de 2008 de la Cour suprême (Riigikohus) n'a pas modifié le plan de développement local qui incluait le territoire d'un bosquet sacré. En revanche, la Cour a, en 2012, mis fin à une licence d'exploitation forestière concernant une forêt jouxtant le bosquet de Maardu. 
tant qu'espaces spirituels. En dépit de l'importance de ces questions, il semble extrêmement difficile, voire impossible, de régler par la loi la protection des espaces sacrés naturels. Où commence et où s'arrête le sacré ? La subjectivité de l'expérience du sacré se prête mal à une régulation juridique et les débats deviennent passionnés, même si les archéologues ont mis en avant que le point de vue sur les sites sacrés défendu par la MTRA a été accepté sans critique par l'opinion publique ${ }^{44}$.

L'intérêt de cette question des sites naturels sacrés est de souligner que la protection du patrimoine religieux ne peut se réduire à sa préservation matérielle et au respect des règles de conservation du patrimoine culturel, mais renvoie au positionnement de l'État, des institutions et des communautés religieuses et à leur autorité dans le paysage spirituel local. 